\title{
Depression, resilience and post traumatic stress disorder (PTSD) in asylum-seeker war refugees
}

\author{
Maria Malliarou \\ University of Thessaly \\ Dimitra Tsapakidi \\ Volos Refugee Camp (Moza) \\ Mairi Gouva \\ Panepistimio loanninon \\ Evaggelia Kotrotsiou \\ University of Thessaly \\ Athanasios Nikolentzos \\ Hellenic Open University \\ Pavlos Sarafis ( $\square$ pavlos.sarafis@cut.ac.cy) \\ Cyprus University of Technology
}

\section{Research}

Keywords: PTSD, Depression, Resilience, Immigrants

Posted Date: March 5th, 2020

DOI: https://doi.org/10.21203/rs.3.rs-16163/v1

License: (c) (i) This work is licensed under a Creative Commons Attribution 4.0 International License.

Read Full License 


\section{Abstract}

Background The number of refugees and displaced people who have been forced to migrate due to war, mass violence and political instability has reached unprecedented levels. The objective of this study was to assess the degree of depression, resilience and post-traumatic stress disorder (PTSD) in asylum-seeker war refugees, temporally stationed in Larisa, Central Greece, through self-administered questionnaire. Simple regression, Pearson's correlation coefficient and one-way Anova was used.

Methods A cross-sectional study carried out with asylum-seeker war refugees residing in Larisa at a number of apartments rented and ran by the UNHCR and the municipality of Larisa. For the data collection PHQ-9 questionnaire, CD-RISC questionnaire and Harvard Trauma questionnaire (HTQ) were used. Descriptive statistics were applied, as well as Cronbach's alpha coefficient.

Results A total of 64 immigrants ( 40 males and 23 females, 1-mssing value) agreed to participate in the research. The mean age was 35.72 (SD 7.45), 35 respondents were classified as increased PTSD (scores $>2.5$ ). The results showed a physically and exhausted sample, with marginal mental strength (Mean = $59.38 \%)$, fluctuating between moderate $(\mathrm{N} \%=28.10 \%)$ and severe depression $(\mathrm{N} \%=28.10 \%)$. About one in two participants $(\mathrm{N} \%=54.70 \%)$ showed signs of post-traumatic stress disorder PTSD, reporting traumatic experiences that mainly involved immediate events of violence, i.e. beating, while there were very few cases of sexual abuse or violence. A key factor in the formulation of PTSD, depression and Mental Stress Indicators was found to be age but limited to 43 years.

Conclusion This study revealed that the refugees of the sample are tired, after being absent and idle for about 2 years from their homeland, in a marginal state of collapse. The most important event-hope that sustains its moderate resilience is the grant of asylum and the hope of arrival in the country of destination.

\section{Background}

The number of refugees and displaced people who have been forced to migrate due to war, mass violence and political instability has reached unprecedented levels. There were an estimated 1.9 million refugees in 1951 and 2.4 million in 1970 worldwide, with dramatic increases to 12.1 million in 2000 and 14.3 million in 2014. These estimates bring in only a fraction of the additional millions of other displaced, asylum and humanitarian aid seekers who cannot return to their home countries [1]. Among refugees, traumatic exposure before and during migration is closely linked to mental health and psychological distress $[2,3]$. Evidence suggest that these symptoms, among former refugees, may persist years after resettlement [4]. Consequently, there is increased acceptance, among the academic community, that postwar post-traumatic stress is affecting family members [5] with potential long-term effects on the health and psychosocial well-being of future generations [6].

Intergenerational trauma generally refers to the ways in which the trauma experienced in one generation affects the health and well-being of offspring of future generations [6, 7]. The negative effects can 
include a number of psychiatric symptoms as well as a greater vulnerability to stress as a result of traumatic experiences in various populations, including survivors of abuse, armed conflict and genocide $[7,8]$. Since refugees are scattered in many geographical places, it has been observed that the investigation of trauma, resilience and well-being of refugees may contain major differences in methodologies and study designs. The present research investigates these characteristics and compares its results with similar surveys and reports. The term immigrant or refugee will be used extensively to refer to people who have been forced to flee their countries of origin in the context of political violence, persecution and instability $[9,10]$.

\section{Methods}

\section{Participants and Procedure}

A facility based cross-sectional study was conducted from March 3, to July 15, 2019 in the town of Larisa at a number of apartments rented and ran by the UNHCR and the public benefit organization of the municipality of Larisa, within context of the implementation of the humanitarian program "Emergency Support To Integration \& Accommodation - ESTIA" which hosts approximately 500 immigrants. As immigrants belong to vulnerable social groups, who experienced difficult and life-threatening situations, the research was conducted through specialized staff in the hospitality area. This way, the negative experiences of immigrants were simply referred to, reducing the risk for research participants to revive these experiences. A great effort was made to an inventory data collection, which aimed to the collection of at least $50 \%$ of the 500 families, which proved to be a daunting task, due to the refusal of the immigrants to participate as well as the extended length of the interview process.

The sample size was 64 participants which was equal to $12.80 \%$ of the total population residing at the designated apartments. Convenience sampling method was implemented as other, more elaborate sampling methods, might return null or significantly lower sample sizes.

\section{Data Analysis}

After collection, data were entered and coded in SPSS V23 statistical software. Excel 2016 was also used for data analysis, and the production of specific graphs. Data analysis included Mann - Whitney, Kruskal - Wallis test for the investigation of statistically significant mean differences, Pearson's correlation coefficient and multiple regression for the investigation of the associations between the produced indexes. Results with $\mathrm{p}$-level $<0.05$ were considered as statistically significant.

Data were collected using a structured self-administered questionnaire translated into the Iraqi language. The extent of a person's depression was measured with the help of PHQ-9 questionnaire [11] which includes 9 questions based on a 4-point Likert scale ( $1=$ Not at all to $4=A$ Almost every day). Resilience was measured with the help of CD-RISC questionnaire [12] which includes a five point Likert scale (1=Not true to $5=$ Almost always true). Additional subscales were used based on Dimitriadou and Stalika [13]. These subscales measured Personal Ability, High Standards \& Endurance (5 items), Confidence (6 items), 
Positive acceptance (7 items), Audit ( 3 items) and spiritual influences ( 2 items). Post-traumatic stress disorder (PTSD) was measured with the help of Harvard Trauma questionnaire (HTQ) [14]. The HTQ questionnaire consists of five parts namely, Trauma events, personal description, head injury, Symptoms and torture history.

\section{Results}

From a total of 500 stationed immigrants 64 participated giving a response rate of $12.80 \%$. The mean age of the respondents was 36 (SD \pm 7.4 ) years. The majority of the respondents $40(63.50 \%)$ were men. Their family status was mostly described as married 58 (92.10\%), having two 22 (34.40\%) or three 20 $(31.30 \%)$ children. From the 10 immigrants who stated having relatives in other countries, 7 cases referred to family members i.e. parents, husband or wife and 3 cases involved children left behind. Regarding to the nationality of the immigrants, the majority were self-reported as Syrians 41 (66.40\%). Other, referred nationalities were Iraqi, Pakistani, Afghanis, Palestinians and Kurdish. Also, the majority of the immigrants had attended first grade $38,(63.30 \%)$ and all immigrants had annual income less than 3,000 Euros.

The average travel time to Greece was approximately 77 days ( $S D \pm 92.89)$, their waiting period until reallocation to the designated apartments was 92 days $(S D \pm 789.13)$, and their mean residence time in the designated apartments was approximately $522(\mathrm{SD} \pm 313.13)$ days. In all cases, there was a large variability with standard deviation approximately equal to the average value and range ranging from 395 days for the time of travel and accommodation to 1525 days for accommodation (Table 1).

None of the immigrants was previously deported or had visited Greece in the past. Living conditions were described as bad $29(46 \%)$ or medium $27(42.90 \%)$ and the relations with the local population as medium $53(84.10 \%)$. Living conditions and relation to the local population were dependent to each other $\left(X^{2}=44.666\right.$, d.f. $\left.=4, p<0.001\right)$, under a weak and positive relation ( $\left.r h o=0.252, p=0.046\right)$.

\section{Depression}

The results of PHQ-9 questionnaire showed that most of the sample was categorized as having low depression 21 (32.80\%). The categories of moderate and severe depression had the same incidence rates $18(28.10 \%)$ whereas the pathological depression category included only 7 cases $(10.90 \%)$.

\section{Resilience}

The sample exhibited moderate resilience as the mean score was below $60 \%$ since mean score value was equal to 59.38 ( $S D \pm 12.75)$. The subscales showed similar values to the overall mean of the questionnaire except for the "Spiritual Effects" subscale. In this case a high average value of $75.38 \%$ was observed.

\section{Trauma}


According to their answers, Forced migration $53(82.81 \%)$, destruction, confiscation or theft of property 46 (71.87\%), oppression due to ethnicity, religion or sect $46(71.87 \%)$, forced internal migration to a lower standard of living 44 (68.75\%), illegal research 43 (67.87\%) and loss of home 49 (76.56\%) were the main traumatic events experienced by the participants. Of the 25 immigrants who described their personal traumatic events in their home country, most reports referred to the bombings in their home country ( $\mathrm{N}=$ $10)$, their journey to Greece $(N=6)$, the loss of their home $(N=4)$. , the separation from their homeland ( $N$ $=4)$ and their torture $(\mathrm{N}=3)$. In addition, individual reports of migrants described threats due to different religion and occupation (hairdresser) that did not conform to Islamic beliefs. These reports also included illegal detention on the grounds of religion and the separation of children due to immigration. More detailed descriptions of the bombing referred bombs, rockets and bullets exploded near or passed beside them and torture with electric shocks and upside-down hanging. Regarding their living conditions, most reports described poor living conditions in Chios camp $(\mathrm{N}=8)$ and in general $(\mathrm{N}=5)$. There was also an individual reference $(\mathrm{N}=1)$ to the feeling of insecurity due to racist attacks by other ethnic groups in the designated apartments, negative feelings from the locals and the difficulty of adapting to living spaces.

According to their answer in the head injury part of the HTQ, most cases reported was famine 13 (20.30\%) with average loss of approximately $14 \mathrm{~kg}$ from 9 reported cases. In these cases, weight loss ranged between 6 and $22 \mathrm{~kg}$. Unconsciousness was mainly presented as a result of head blows and a 3minute case of unconsciousness was reported. Choking was reported 3 cases, and the unconsciousness time loss was 2.30 and 120 minutes. PTSD syndrome was found in 35 cases $(54.70 \%)$ and the most frequent tortures that reported were sleep deprivation 39 (60.93\%), exposure to rain or cold $36(56.25 \%)$, exposure to continuous and intense noise $35(54.68 \%)$, exposure to excessive heat and intense sunshine or bright light 35 (54.68\%).

\section{Association between indexes}

Index PHQ-9 was correlated positively with PTSD index $(p=0.652, p<0.001)$ and negative with CDRISC ( $p$ $=-0.256, p=0.041)$. PTSD index was negatively correlated with CDRISC index $(p=-0.443, p<0.001)$. Multiple regression revealed that depression acts as an aggravating factor, while resilience reduces trauma symptoms with the help of a statistically significant model $(F=31.301$, d.f. $=2,63, p<0.001)$, of medium interpretability $\left(R^{2}=0,506\right)$. Age was found as a depression reducing factor but under a statistically significant model $(F=4.961$, d.f. $=1,49, p=0.031)$, with low interpretability $\left(R^{2}=0,094\right)$.

\section{Discussion}

The results of the CD RISC questionnaire showed moderate persistence as the mean score was below $60 \%$, although "Spiritual Effects" subscale score was near $75.50 \%$ indicating immigrants resilience is based on expectations from divine intervention, showing the strong influence of religion, in agreement to Neftçi \& Çetrez [15] and Hatfield et al., [16] results. The reported trauma events of the immigrants did not mention cases of sexual abuse, abduction or hostage in contrast to Arnetz et al., [17] and Neftçi \& Çetrez [15] both to the severity and frequency of these events. 
Men revealed higher mental strengths than women (CD RISC: Men = 62, Women = 55) in agreement with Vukčević, Momirović \& Purić [18] who stated that the reason for this difference was the high levels of stress and anxiety in women. It was also observed that immigrants with no grammar knowledge presented lower mean CD RISC scores (46.28) and higher symptom ratings 2.77 presenting similar qualitative results to those of Hosseini et al., [19].

Examination of the contribution of the PHQ and CD RISC variables to PTSD showed that PTSD symptoms are expected to decrease with increasing resilience scores. Depression has an increasing effect on experiencing PSTD symptoms as higher depression rates are expected to result higher PTSD scores. In addition, it has been observed that age is a reducing factor in PTSD symptoms inductively as it is a factor in lowering PHQ-9 scores in agreement with Vukčević, Momirović \& Purić [18] and other studies $[20,21]$. These results were expected as it was observed that all three variables PHQ-9, CD-RISC and PTSD were correlated.

PHQ-9 scores were correlated positively with PTSD $(p=0.652, p<0.001)$ and negative with CD RISC ( $p=$ $-0.256, p=0.041)$ and PTSD is negatively correlated with CDRISC $(p=-0.443, p<0.001)$. Thus, it becomes clear again that an increase in the degree of depression is expected to result in an increase in the degree of PTSD symptoms as well as a decrease in the immigrant's mental strength. It has also been observed that an increase in the level of mental strength of the immigrant is expected to result in a decrease in the severity of the symptoms.

In conclusion this study revealed that the immigrants of the sample are tired, after being absent and idle for about 2 years from their homeland, in a marginal state of collapse. The most important event-hope that sustains its moderate resilience is the grant of asylum and the hope of arrival in the country of destination.

\section{Conclusions}

Even if war is not considered as the main cause for forced migration, it is still considered one of the most violent forms of forced migration with one of the most severe effects on the psychosynthesis of a refugee. The refugee having experienced the violence from the beginning of its move, as a cause of its move, possibly will continue to experience violence during its move but also upon arrival on its destination or its host country. This study confirmed that the refugees of the sample are tired, after being absent and idle for about 2 years from their homeland and in a marginal state of collapse. However, in terms of resilience it seems that the most important event-hope that sustains its moderate level is the grant of asylum and the hope of arrival in the country of destination.

\section{Study Limitations}

There have been cases of confusion of immigrants either due to the inability to understand the questionnaire or because of confusion between the difficulties encountered during their transition to 
Greece and man-based torture. Although both cases are related to and affect their psychosynthesis, they nevertheless influence the accuracy of the results. Therefore, the participants should have been screened under the details of their asylum application where it is clear or not if they have been war victims. It was also suggested to prepare the sample at the Harvard Trauma Questionnaire before completing it.

\section{Abbreviations}

PTSD

Post-Traumatic Stress Disorder

PHQ-9

Patient Health Questionnaire-9

CD RISC

Connor-Davidson Resilience Scale

HTQ

Harvard Trauma questionnaire

UNHCR

United Nations High Commissioner on Refugees

\section{Declarations}

\section{Ethics approval and consent to participate}

Ethical clearance was obtained from the Municipality of Larisa and the board of trustees of the public benefit organization which provides accommodation to refugees (Reference Number 220/ 08.02.2019).

The researchers were granted permission under the obligation to share the results of the study, protect the anonymity of the participants and inform them about the purpose of the study. In addition, questionnaires were approved by its social workers, while informed written consent and assent were obtained by all the participants who were all over the age of 18 .

\section{Consent for publication}

Not applicable.

\section{Availability of data and materials}

The datasets generated and analyzed during the current study are not publicly available to preserve the privacy of the participants but are available from the corresponding author on reasonable request.

\section{Competing interests}

The authors would like to declare that they have no competing interests.

\section{Funding}


Not applicable

\section{Authors' contributions}

TD collected the data and MM wrote the paper. GM, KE, NA and PS read and commented on the final manuscript. All the authors finally approved the final manuscript.

\section{Acknowledgements}

We would like to thank the participants for their cooperation.

\section{References}

1. United Nations High Commissioner on Refugees (UNHCR): population statistics. UNCHR; 2015. Available from http://www.unhcr.org.

2. Fazel M, Reed RV, Panter-Brick C, Stein A. Mental health of displaced and refugee children resettled in high-income countries: risk and protective factors. Lancet. 2012; 379(9812):266-82.

3. Kirmayer LJ, Narasiah L, Munoz M, Rashid M, Ryder AG, Guzder J, Hassan G, Rousseau C, Pottie K. Common mental health problems in immigrants and refugees: general approach in primary care. Can Med Assoc J. 2011;183(12):E959-E967.

4. Marshall GN, Schell TL, Elliott MN, Berthold SM, Chun CA. Mental health of Cambodian refugees 2 decades after resettlement in the United States. JAMA. 2005; 294(5):571-9.

5. Weine S, Muzurovic N, Kulauzovic Y, Besic S, Lezic A, Mujagic A, Muzurovic J, Spahovic D, Feetham S, Ware N, Knafl K, Pavkovic I. Family consequences of refugee trauma. Fam Process. 2004; 43(2):147-60.

6. Dekel $\mathrm{R}$, Goldblatt $\mathrm{H}$. Is there intergenerational transmission of trauma? The case of combat veterans' children. Am J Orthopsychiatry. 2008; 78(3):281.

7. Bezo B, Maggi S. Living in "survival mode:" Intergenerational transmission of trauma from the Holodomor genocide of 1932-1933 in Ukraine. Soc Sci Med. 2015; 134:87-94.

8. Han M. Relationship among perceived parental trauma, parental attachment, and sense of coherence in Southeast Asian American college students. J Fam Soc Work. 2005; 9(2):25-45.

9. George M. A theoretical understanding of refugee trauma. Clin Soc Work J. 2010; 38(4):379-87.

10. Hein J. Refugees, immigrants, and the state. Annu Rev Sociol. 1993; 43-59.

11. Kroenke K. Spitzer R.L. Williams J.B. The PHQ-9: validity of a brief depression severity measure. Journal of general internal medicine. 2001;16(9), 606-13.

12. Connor KM, Davidson JRT. Development of a new resilience scale: the Connor-Davidson Resilience Scale (CD-RISC). Depression and Anxiety, 2003: 18: 71-82.

13. Dimitriadou, D. Stalikas A. Connor-Davidson Resilience Scale (CD-RISC). Psychometric Instruments in Greece (2nd ed., p. 717). Athens, 2012, Pedio. 


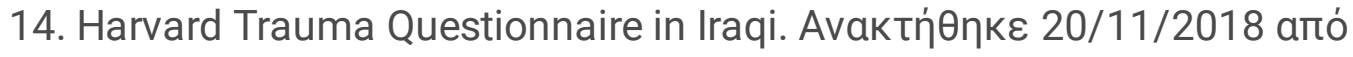
https://www.healtorture.org/sites/healtorture.org/files/4\%20HTQ\%20Arabic.pdf

15. Neftçi NB, Çetrez ÖA. Resilience and Mental Health Risks among Syrian Refugees in Europe: A Cultural Perspective. Acta Psychopathol. 2017;3:65. doi: 10.4172/2469-6676.100137

16. Hatfield B, Mohamad H, Rahim Z, Tanweer H. Mental health and the Asian communities: a local survey $\mathrm{Br} \mathrm{J}$ of Social Work. 1996;26: 315-336

17. Arnetz J, Rofa Y, Arnetz B, Ventimiglia M, Jamil H. Resilience as a protective factor against the development of psychopathology among refugees. J Nerv Ment Dis. 2013;201(3):167-172. doi:10.1097/NMD.0b013e3182848afe

18. Vukčević, M., Momirović, J., Purić, D.. Adaptation of Harvard Trauma Questionnaire for working with refugees and asylum seekers in Serbia. Psihologija, 2016;49(3):277-299.

19. Ashraf Hosseini, Ritsuko Kakuma, Mehdi Ghazinour, Melanie Davern, William. P. Evans \& Harry Minas. Migration experience, resilience and depression: a study of Iranian immigrants living in Australia, International Journal of Culture and Mental Health. 2017; 10:1, 108-120, DOI: 10.1080/17542863.2016.1270977

20. Gerritsen A.A.M. Bramsen, I. Devillé, van Willigen, L H.M. Hovens, J.E. van der Ploeg H.M. Physical and mental health of Afghan, Iranian and Somali asylum seekers and refugees living in the Netherlands. Social Psychiatry and Psychiatric Epidemiology. 2006; 41(1), 18-26. doi:10.1007/s00127-005-0003-5

21. Renner Salem I. Post-traumatic stress in asylum seekers and refugees from Chechnya, Afghanistan, and West Africa: gender differences in symptomatology and coping. The International Journal of Social Psychiatry. 2009;55(2), 99-108. doi:10.1177/0020764008092341

\section{Tables}

Table 1 Time of travel, reallocation and residence $(n=64)$

\begin{tabular}{lcccccc}
\hline \hline & Mean & Median & S.D. & Range & Min & Max \\
\hline Days of travel Greece & 77.73 & 61 & 92.895 & 393 & 2 & 395 \\
Stand by time in days & 91.73 & 62 & 89.126 & 394 & 1 & 395 \\
Days of residence in the designated apartments & 521.94 & 513 & 313.132 & 1486 & 39 & 1525 \\
\hline \hline
\end{tabular}

